\title{
BMJ Open Statin therapy for patients with aortic stenosis who underwent transcatheter aortic valve implantation: a report from a Japanese multicentre registry
}

Fumiaki Yashima (D) , ${ }^{1,2}$ Masahiko Hara, ${ }^{3}$ Taku Inohara, ${ }^{4}$ Masahiro Jinzaki, ${ }^{5}$ Hideyuki Shimizu, ${ }^{6}$ Keiichi Fukuda, ${ }^{2}$ Makoto Tanaka, ${ }^{2}$ Masanori Yamamoto, ${ }^{7,8}$ Yusuke Watanabe, ${ }^{9}$ Toru Naganuma, ${ }^{10}$ Shinichi Shirai, ${ }^{11}$ Masahiro Yamawaki, ${ }^{12}$ Norio Tada, ${ }^{13}$ Futoshi Yamanaka, ${ }^{14}$ Kazuki Mizutani, ${ }^{15}$ Hiroshi Ueno, ${ }^{16}$ Minoru Tabata, ${ }^{17}$ Kensuke Takagi, ${ }^{18}$ Kentaro Hayashida, ${ }^{2}$ OCEAN-TAVI registry

To cite: Yashima F, Hara M, Inohara T, et al. Statin therapy for patients with aortic stenosis who underwent transcatheter aortic valve implantation: a report from a Japanese multicentre registry. BMJ Open 2021;11:e044319. doi:10.1136/ bmjopen-2020-044319

- Prepublication history and additional supplemental material for this paper are available online. To view these files, please visit the journal online (http://dx.doi.org/10.1136/ bmjopen-2020-044319).

Received 08 September 2020 Accepted 19 May 2021

Check for updates

(c) Author(s) (or their employer(s)) 2021. Re-use permitted under CC BY-NC. No commercial re-use. See rights and permissions. Published by BMJ.

For numbered affiliations see end of article.

Correspondence to Dr Fumiaki Yashima; yashima.fumiaki@gmail.com

\section{ABSTRACT}

Objective Data on statin for patients with aortic stenosis (AS) who underwent transcatheter aortic valve implantation (TAVI) are limited. The present study aimed to evaluate the impact of statin on midterm mortality of TAVI patients.

Design Observational study.

Setting This study included patients with AS from a Japanese multicentre registry who underwent TAVI. Participants The overall cohort included 2588 patients (84.4 \pm 5.2 years); majority were women $(69.3 \%)$. The Society of Thoracic Surgeons risk score was $6.55 \%$ (IQR 4.55\%-9.50\%), the Euro II score was 3.74\% (IQR 2.34\%$6.02 \%$ ) and the Clinical Frailty Scale score was $3.9 \pm 1.2$. Interventions We classified patients based on statin at admission and identified 936 matched pairs after propensity score matching.

Primary and secondary outcome measures The outcomes were all-cause and cardiovascular mortality. Results The median follow-up was 660 days. Statin at admission was associated with a significant reduction in all-cause mortality (adjusted HR (aHR) 0.76, 95\% Cl 0.58 to $0.99, p=0.04$ ) and cardiovascular mortality (aHR 0.64 , $95 \% \mathrm{Cl} 0.42$ to $0.97, p=0.04$ ). In the octogenarians, statin was associated with significantly lower all-cause mortality (aHR 0.87, 95\% Cl 0.75 to $0.99, p=0.04$ ); however, the impact in the nonagenarians appeared to be lower (aHR $0.84,95 \% \mathrm{Cl} 0.62$ to $1.13, \mathrm{p}=0.25)$. Comparing four groups according to previous coronary artery disease (CAD) and statin, there was a significant difference in all-cause mortality, and patients who did not receive statin despite previous CAD showed the worst prognosis (aHR 1.33, $95 \% \mathrm{Cl} 1.12$ to 1.57 (patients who received statin without previous CAD as a reference), $p<0.01$ ).

Conclusions Statin for TAVI patients will be beneficial even in octogenarians, but the benefits may disappear in nonagenarians. In addition, statin will be essential for TAVI patients with CAD. Further research is warranted to confirm and generalise our findings since this study has the inherent limitations of an observational study and included only Japanese patients.

Trial registration number UMIN000020423.

\section{Strengths and limitations of this study}

- The present study includes the largest number of patients with aortic stenosis who underwent transcatheter aortic valve implantation, assessing the impact of statin therapy on midterm all-cause and cardiovascular mortality.

- This is the first study to investigate the difference in statin effect among octogenarians and nonagenarians and to evaluate how the impact of statin therapy differed according to the underlying coronary artery disease.

- All-cause and cardiovascular mortality were analysed using propensity score matching and Cox proportional hazards regression model.

- Unknown and unmeasurable factors may have confounded the relationship between statin therapy at admission and mortality due to the nature of an observational study.

- We could not assess intolerance in patients eligible for statin treatment but who could not continue treatment due to statin side effects.

\section{INTRODUCTION}

Transcatheter aortic valve implantation (TAVI) is an established treatment for severe aortic stenosis (AS) ${ }^{1-4}$ However, long-term survival after TAVI is not satisfactory, as shown in a meta-analysis including 31 studies, where the 5 -year and 7-year survival rates were $48 \%$ and $28 \%$, respectively. ${ }^{5}$ TAVI patients are very elderly and have many cardiovascular comorbidities, such as coronary artery disease (CAD), stroke and peripheral artery disease (PAD). ${ }^{126}$ Therefore, adjunctive optimal medical therapy is required to improve prognosis after TAVI. Statin therapy is expected to reduce cardiovascular risk and mortality in patients who have undergone TAVI; however, data on statin therapy for TAVI patients are limited. A 


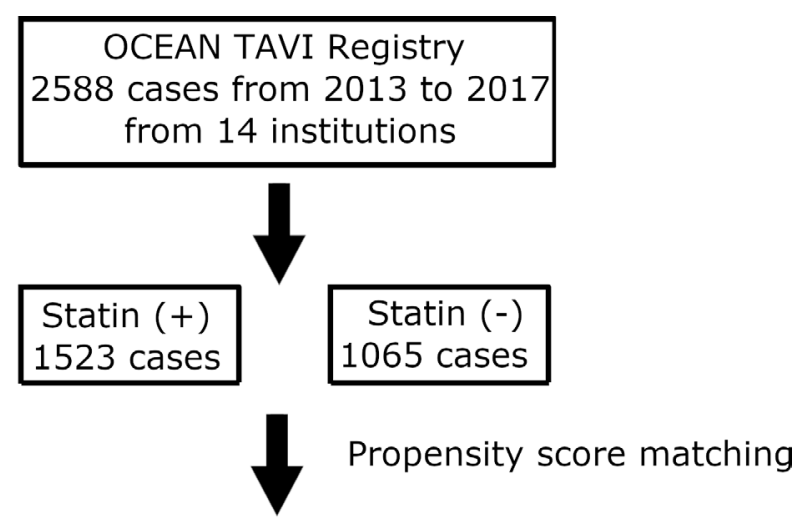

\begin{tabular}{|l|}
\hline Statin $(+)$ \\
936 cases
\end{tabular}$\quad \begin{array}{r}\text { Statin }(-) \\
936 \text { cases }\end{array}$

Figure 1 Flow chart of patient selection for the present study. OCEAN, Optimized CathEter vAlvular iNtervention; TAVI, transcatheter aortic valve implantation.

report from the Placement of Aortic Transcatheter Valve II (PARTNER II) and Sapien 3 clinical trials or associated registries showed that statin therapy was associated with a lower 2-year mortality rate compared with patients not on statin therapy. ${ }^{7}$ However, the study did not demonstrate any differences in statin effect among octogenarians and nonagenarians and did not evaluate whether the impact of statin therapy would differ according to the underlying CAD. Therefore, the present study aimed to evaluate the impact of statin therapy on midterm mortality of TAVI patients and its association with age or the underlying $\mathrm{CAD}$, using our Japanese multicentre registry data.

\section{METHODS}

\section{Study population and design}

All patients with severe AS who underwent TAVI at 14 Japanese centres (Keio University Hospital, Teikyo University Hospital, Toyohashi Heart Center, Nagoya Heart Center, New Tokyo Hospital, Kokura Memorial Hospital, Saiseikai Yokohama City Eastern Hospital, Sendai Kousei Hospital, Shonan Kamakura General Hospital, Osaka City University Graduate School of Medicine, Kishiwada Tokushukai Hospital, Toyama University Hospital, Tokyo Bay Urayasu Ichikawa Medical Center and Ogaki Municipal Hospital) between 2013 and 2017 were prospectively included in our TAVI registry (Optimized CathEter vAlvular iNtervention (OCEAN-TAVI) registry). ${ }^{8-10}$ Informed consent was obtained from all patients. The OCEAN-TAVI registry was registered with the University Hospital Medical Information Network Clinical Trial Registry and accepted by the International Committee of Medical Journal Editors.

Patients received transcatheter heart valves (THVs) via either the transfemoral, transapical or transaortic approach. Sapien XT valves, Sapien 3 valves (Edwards Lifesciences, Irvine, California), CoreValve and Evolut R (Medtronic, Minneapolis, Minnesota) were used as THVs. A total of 2588 patients were treated with TAVI between 2013 and
2017. They were categorised into two groups according to statin administration at admission for TAVI procedures (figure 1). We set the primary endpoint as midterm allcause mortality for up to 3 years. The secondary endpoints included midterm cardiovascular mortality, midterm noncardiovascular mortality up to 3 years and 30-day all-cause mortality.

We performed propensity score (PS) matching, as described below, and compared the endpoints between the two groups in the matched cohort. In addition, we categorised the matched cohort into two cohorts, an octogenarian cohort (80-89 years old) and a nonagenarian cohort (90 years or older), and investigated differences by age in the impact of statin on midterm all-cause mortality. Furthermore, we classified the overall cohort into four groups according to history of CAD and statin treatment at admission and evaluated whether the impact of statin differed according to the underlying CAD condition. We also explored predictors of midterm all-cause mortality in the overall cohort using multivariate analysis.

Clinical outcomes including all-cause mortality and cardiovascular mortality were defined according to the Valve Academic Research Consortium-2 consensus document. ${ }^{11}$

\section{Statistical analysis}

Continuous variables are expressed as mean $\pm \mathrm{SD}$ and categorical variables are expressed as percentages. Continuous variables were compared using the Wilcoxon rank-sum test. The $\chi^{2}$ test was used to compare categorical variables. Survival curves up to 3 years were presented as KaplanMeier curves, and the log-rank test was used for comparison of the statin and non-statin groups. Cox proportional hazards regression analyses were performed to identify independent correlates for midterm all-cause mortality.

PS matching ${ }^{12}{ }^{13}$ was used to account for differences in baseline characteristics. PS was calculated for each patient using a logistic regression model to predict stratification into the statin group based on the following variables: age; sex; body surface area; smoking; diabetes; hypertension; history of $\mathrm{CAD}$, myocardial infarction (MI), percutaneous coronary intervention, coronary artery bypass grafting, stroke and PAD; atrial fibrillation; estimated glomerular filtration rate; haemoglobin level; renin-angiotensin inhibitor treatment at admission; New York Heart Association (NYHA) class 3 or 4; Clinical Frailty Scale ${ }^{8}$; and Society of Thoracic Surgeons (STS) risk score. PS matching was performed using 1:1 matching without replacement, with the calliper width equal to $0.2 \mathrm{SD}$ of the PS logit. The balance between the statin and non-statin groups in the matched cohort was estimated using absolute standardised difference. Cox proportional hazards analyses were performed to assess the impact of statin on clinical outcomes. In addition to the PS matching model, we built a multivariable model by inverse probability of treatment weighting (IPTW) using PS. ${ }^{14}$ All reported $p$ values were two-sided, and a $p$ value $<0.05$ was considered statistically significant. All statistical analyses were performed using the $\mathrm{R}$ software package (V.3.3.2; $\mathrm{R}$ Development Core Team, Vienna, Austria). 


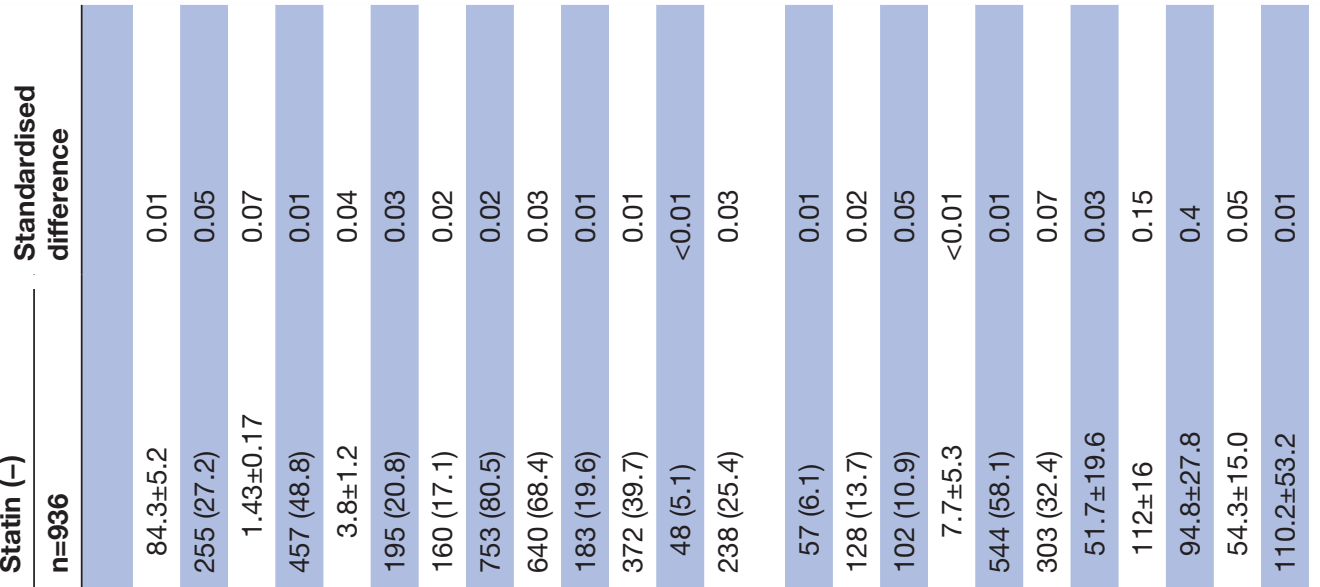

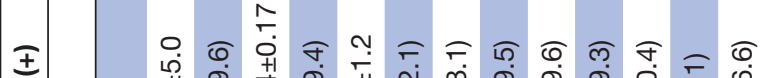

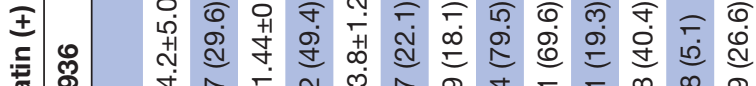

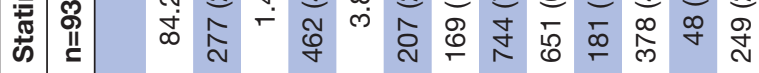

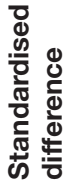

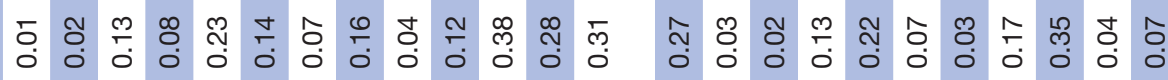

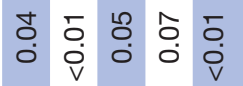

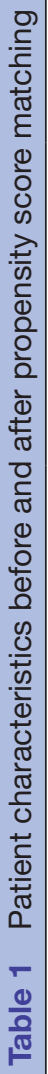

$\frac{2}{\frac{2}{5}}$

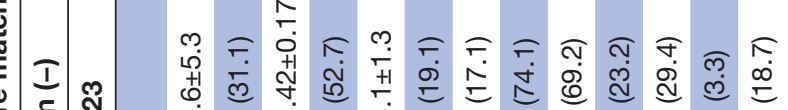

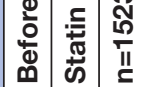

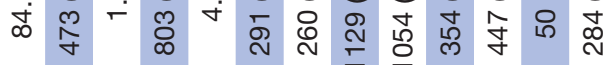

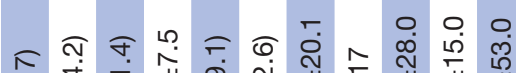

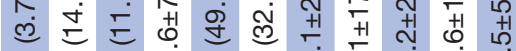

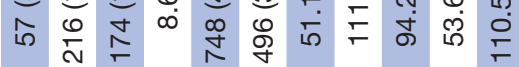

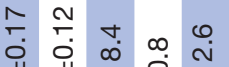

H

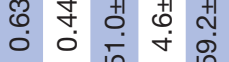

I

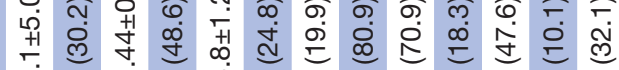

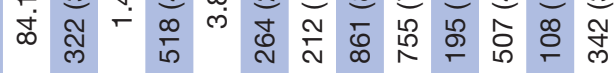

กิำ

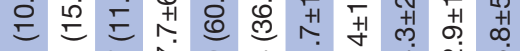

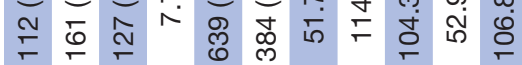

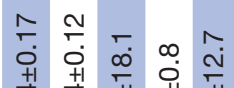

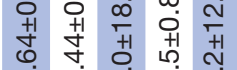

o 0 i⿱

$\frac{8}{8}$

N N

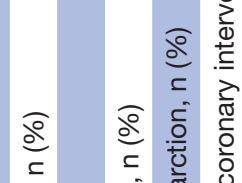

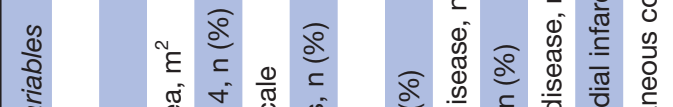

至

$\frac{\sqrt{2}}{2}$

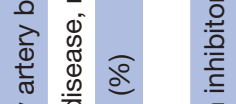

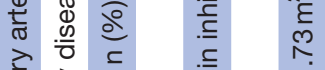

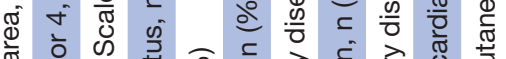

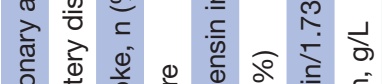

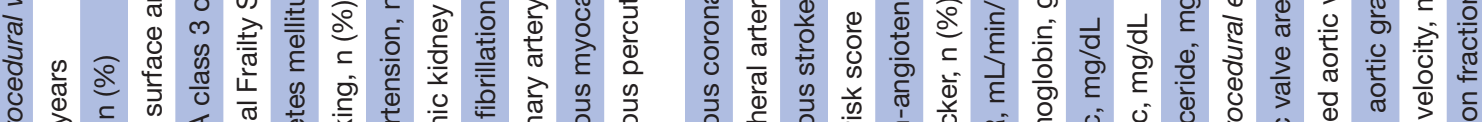

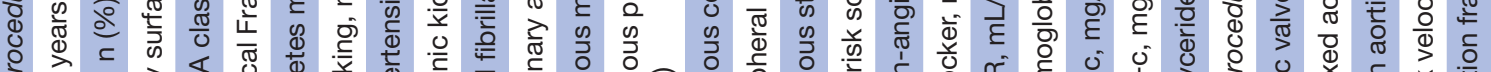

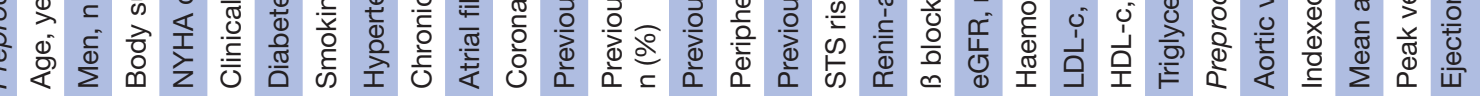




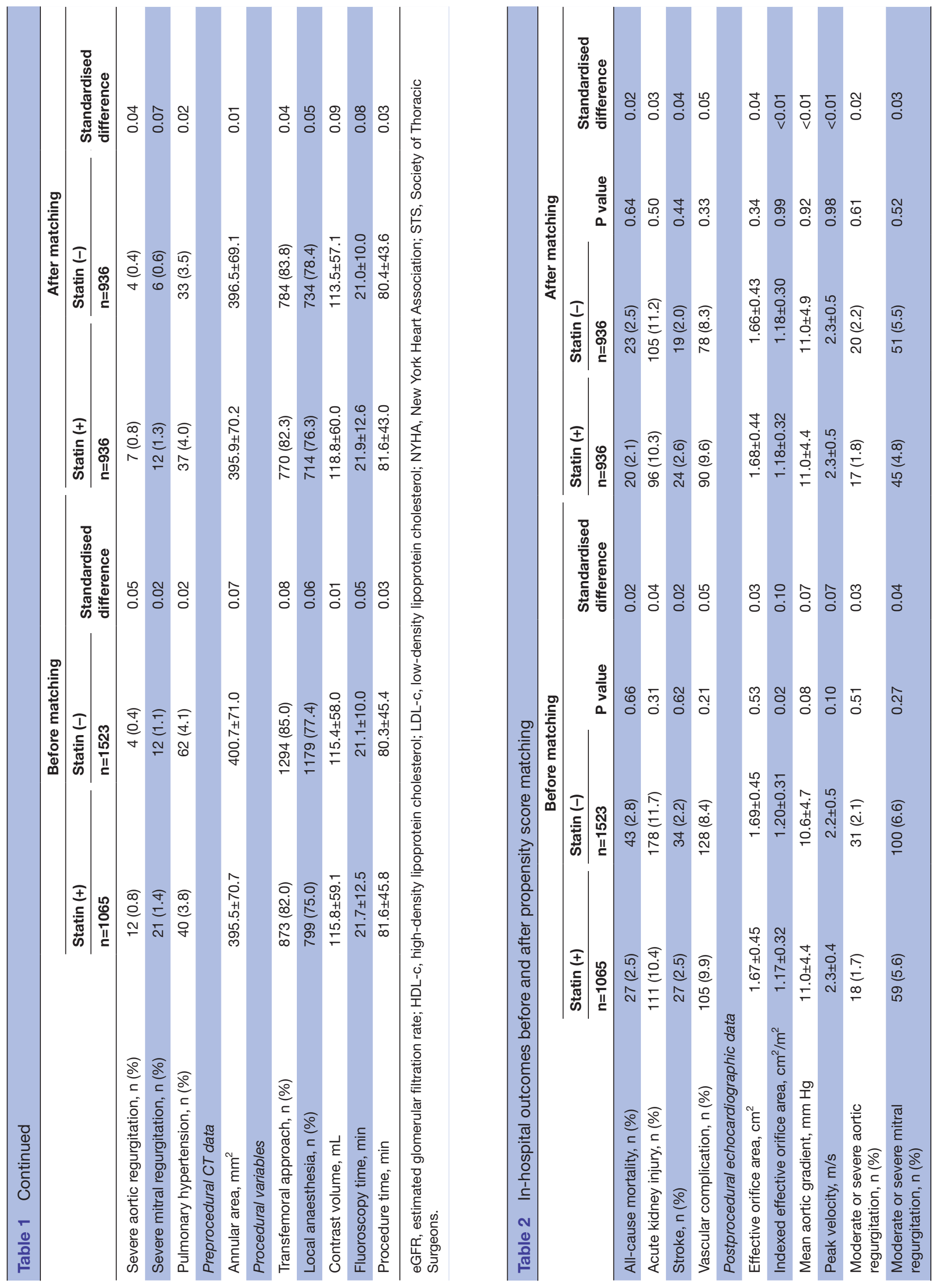

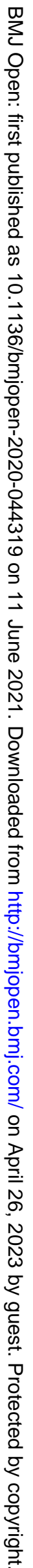


A
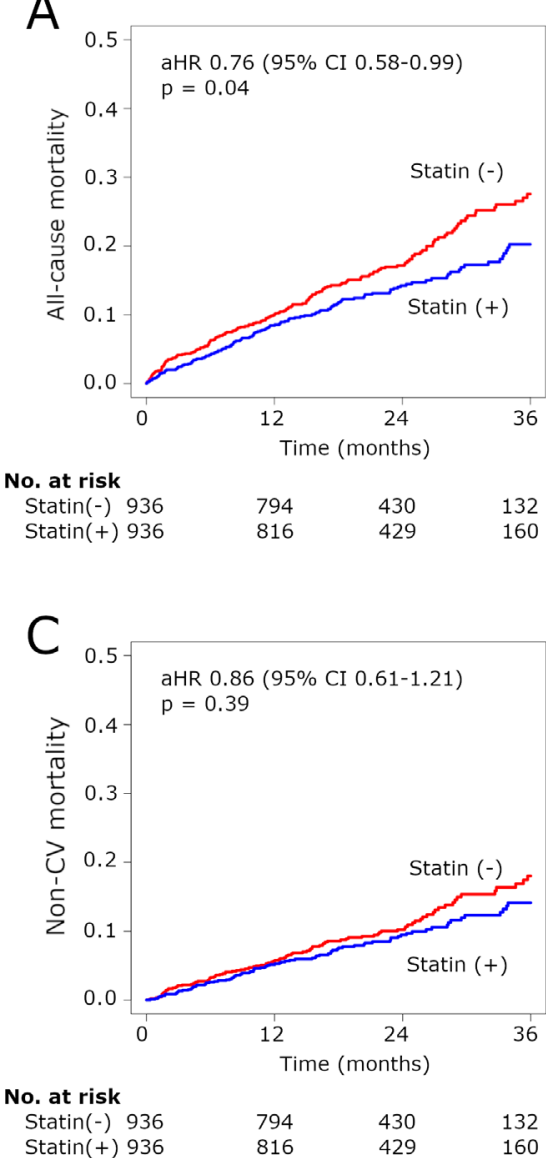

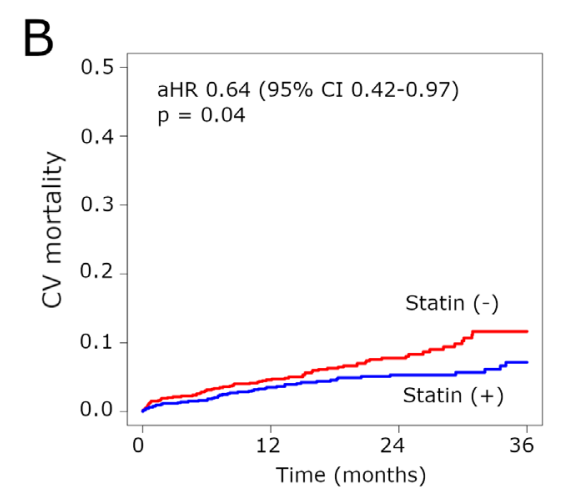

No. at risk

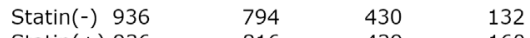
$\begin{array}{llll}\text { Statin }(+) 936 & 816 & 429 & 160\end{array}$

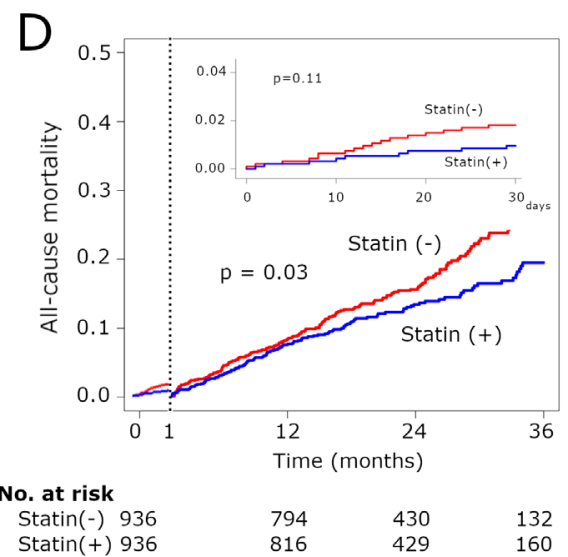

Figure 2 Kaplan-Meier curves for midterm and 30-day mortality in the matched cohort. Kaplan-Meier curves for midterm all-cause mortality (A), CV mortality (B) and non-CV mortality (C) in the matched cohort. (D) Kaplan-Meier curve for 30-day allcause mortality and midterm all-cause mortality with the landmark analysis from 30 days in the matched cohort. aHR, adjusted $\mathrm{HR}$; CV, cardiovascular.

\section{Patient and public involvement statement}

Patients were first involved in the research when they underwent TAVI and registered to the OCEAN-TAVI registry through a web-based data collection system. Research questions and outcome measures were developed by the OCEAN-TAVI registry investigators. Patients were informed about the registration. They were asked to assess the burden of the intervention and the time required to participate in the research. Information on the registry and the study results are available on the website of the OCEANTAVI registry.

\section{RESULTS}

Among the 2588 patients who underwent TAVI, 1523 and 1065 patients were classified into the statin and the non-statin group, respectively (figure 1). The distribution of PS in the statin and non-statin groups is shown in online supplemental figure S1. After 1:1 PS matching, we identified 936 matched pairs of patients with similar PS. The patient characteristics of the statin and non-statin groups before and after matching are summarised in table 1 . The overall cohort included very elderly patients $(84.4 \pm 5.2$ years $)$. The majority of the cohort were female $(69.3 \%)$. The STS risk score was $6.55 \%$ (IQR $4.55 \%-9.50 \%$ ), the Euro II score was $3.74 \%$ (IQR 2.34\%-6.02\%) and the Clinical Frailty Scale score was $3.9 \pm 1.2$.

After PS matching, the two groups were well balanced in terms of preprocedural patient characteristics and procedural variables. In-hospital all-cause mortality, acute kidney injury, stroke and vascular complications did not differ between the two groups. Postprocedural echocardiography data showed no significant differences between the two groups (table 2). The patient characteristics and in-hospital outcomes of the PS matched and unmatched groups are summarised in online supplemental tables S1 and S2. There were several differences between the two groups. The proportion of male patients was lower in the PS matched group than in the unmatched group (532 $(28.4 \%)$ vs $263(36.7 \%), \mathrm{p}<0.01)$, NYHA class 3 or 4 was less frequent in the matched group (919 (49.1\%) vs $402(56.2 \%), \mathrm{p}<0.01)$, the Clinical Frailty Scale score was lower in the matched group $(3.8 \pm 1.2$ vs $4.2 \pm 1.4$, $\mathrm{p}<0.01$ ), and the STS risk score was lower in the matched 

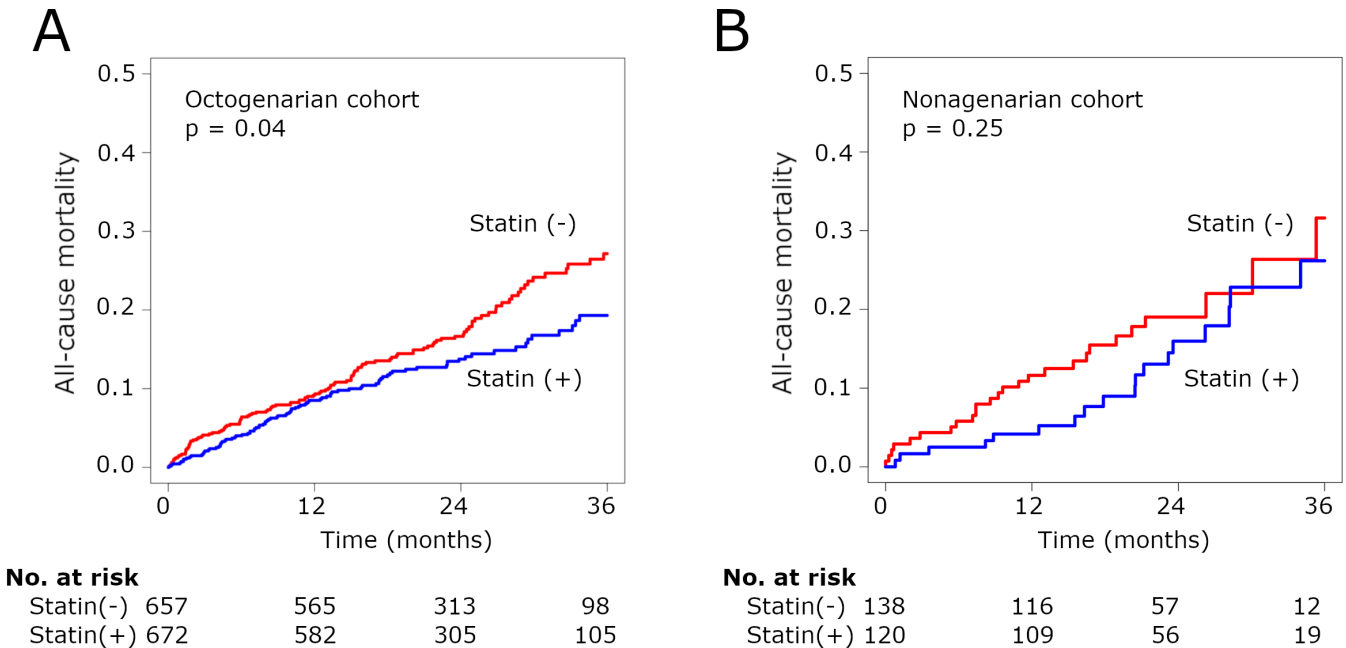

Figure 3 Kaplan-Meier curves for all-cause mortality in the octogenarian (A) and nonagenarian (B) cohorts.

group (7.7 \pm 5.7 vs $9.8 \pm 9.2, \mathrm{p}<0.01)$. History of CAD was more frequent $(750(40.1 \%)$ vs $204(28.5 \%), \mathrm{p}<0.01)$ but previous MI was less frequent (96 (5.1\%) vs $62(8.7 \%)$, $\mathrm{p}<0.01)$ in the PS matched group. In-hospital all-cause mortality $(43(2.3 \%)$ vs $27(3.8 \%), \mathrm{p}<0.01)$ and bleeding (421 $(22.5 \%)$ vs $199(27.8 \%), \mathrm{p}<0.01)$ were lower in the matched group than in the unmatched group.

In the overall cohort, the median follow-up period was 660 days. Statin therapy was associated with significantly lower midterm all-cause mortality in the PS matched cohort (adjusted HR (aHR) $0.76,95 \%$ CI 0.58 to 0.99 , $\mathrm{p}=0.04$ ) (figure 2A), which was consistent with the IPTW model (aHR $0.80,95 \%$ CI 0.65 to $0.99, \mathrm{p}=0.04$ ). The

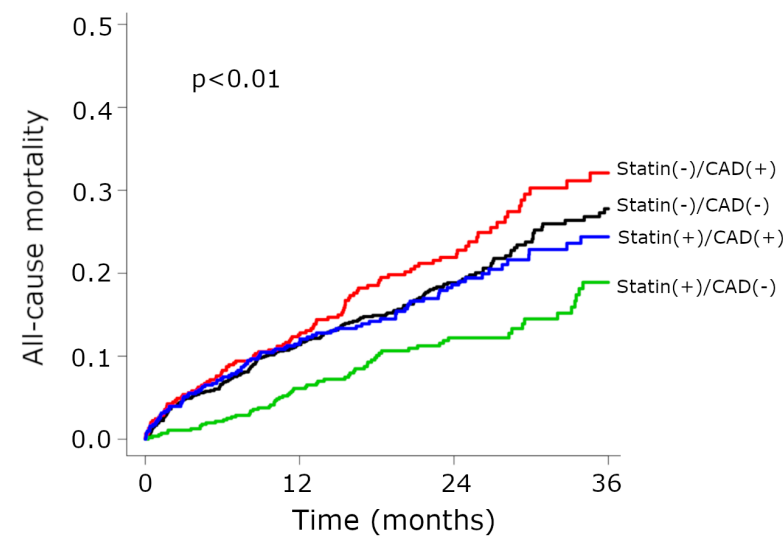

No. at risk

Statin(-)/CAD(+) 447

Statin(-)/CAD(-) 1076

Statin(+)/CAD(+) 507

Statin $(+) / \operatorname{CAD}(-) 558$

$\begin{array}{lll}368 & 201 & 59 \\ 895 & 456 & 142 \\ 428 & 231 & 87 \\ 497 & 256 & 93\end{array}$

Figure 4 Kaplan-Meier curve for all-cause mortality according to previous coronary artery disease (CAD) and statin therapy in the overall cohort.
Kaplan-Meier curves relative to the midterm outcomes additionally showed a significant difference in cardiovascular mortality (aHR $0.64,95 \%$ CI 0.42 to $0.97, \mathrm{p}=0.04$ ) and an insignificant difference in non-cardiovascular mortality (aHR $0.86,95 \%$ CI 0.61 to $1.21, \mathrm{p}=0.39$ ) between the two groups (figure 2B,C). There was no significant difference in 30-day all-cause mortality (aHR 0.73, 95\% CI 0.47 to $1.08, \mathrm{p}=0.11$ ), and a landmark analysis after 30 days showed a significant difference in midterm allcause mortality (aHR $0.88,95 \%$ CI 0.78 to $0.99, \mathrm{p}=0.03$ ) (figure 2D).

In the octogenarian cohort (80-89 years old), statin therapy was associated with significantly lower midterm all-cause mortality (aHR $0.87,95 \%$ CI 0.75 to 0.99 , $\mathrm{p}=0.04$ ) (figure 3A), but the impact in the nonagenarian cohort (90 years or older) appeared to be lower (aHR 0.84, $95 \% \mathrm{CI} 0.62$ to $1.13, \mathrm{p}=0.25$ ) (figure $3 \mathrm{~B}$ ). The $\mathrm{p}$ value for interaction was 0.90 .

Furthermore, comparing the four groups according to previous CAD and statin therapy, there was a significant difference in midterm all-cause mortality $(\mathrm{p}<0.01)$ (figure 4). Patients who did not receive statin therapy despite a history of CAD showed the worst prognosis (aHR 1.33, 95\% CI 1.12 to 1.57; patients who received statin without previous $\mathrm{CAD}$ as a reference). Their survival curve diverged from that of the patients without previous CAD or statin after 1 year. In addition, patients with previous CAD and statin therapy (aHR 1.04, 95\% CI 0.87 to 1.23 ) seemed to obtain similar risks with those who did not have previous CAD or statin therapy (aHR 1.11, 95\% CI 0.96 to 1.27; patients who received statin without previous $\mathrm{CAD}$ as a reference).

The results of the univariate and multivariate Cox proportional hazards regression analyses are shown in table 3. Statin therapy at admission was independently associated with lower all-cause mortality (aHR 0.86, $95 \%$ CI 0.77 to $0.95, \mathrm{p}<0.01)$. 
Table 3 Univariate and multivariate Cox proportional regression analyses of all-cause mortality in the overall cohort

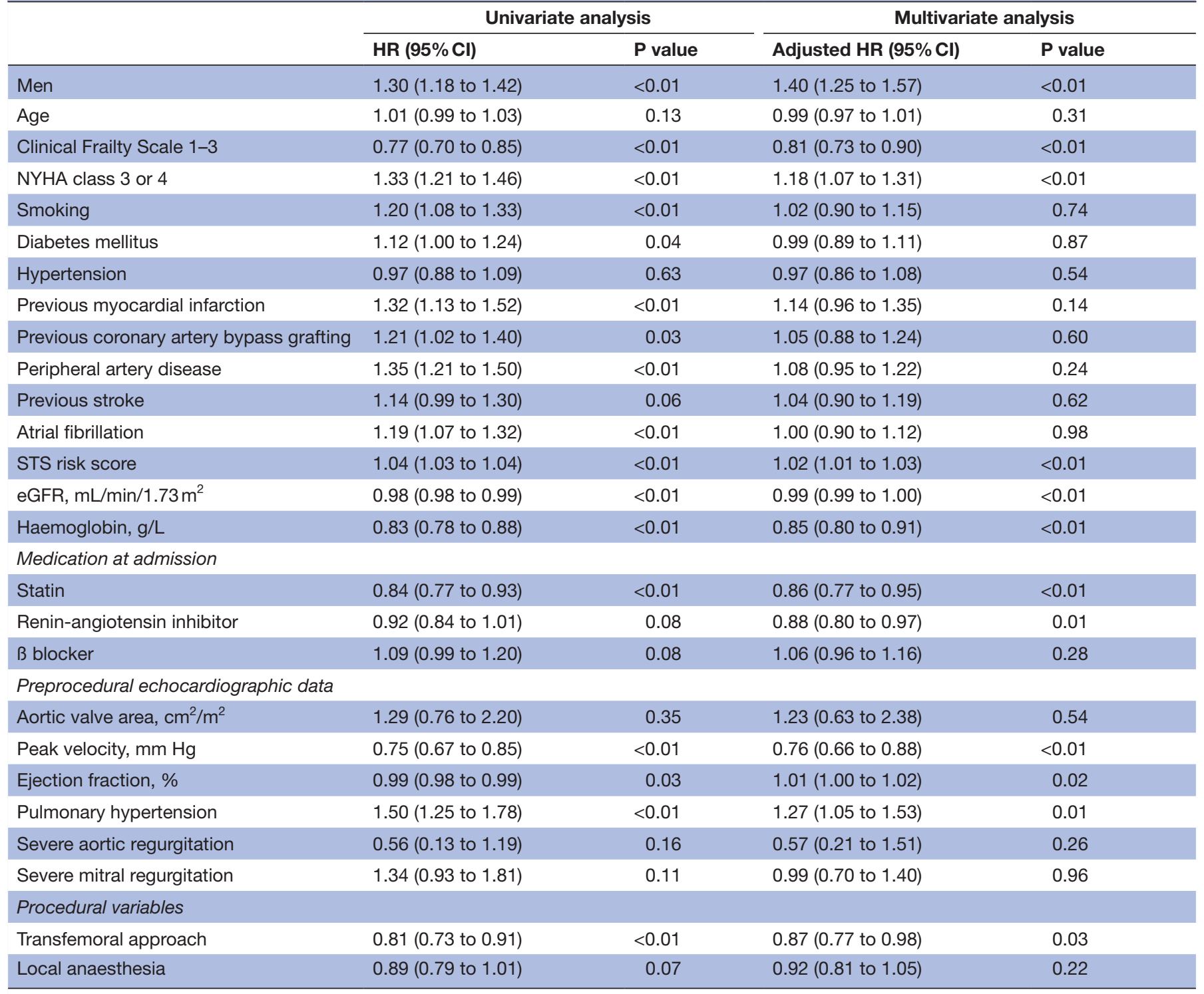

eGFR, estimated glomerular filtration rate; NYHA, New York Heart Association; STS, Society of Thoracic Surgeons.

\section{DISCUSSION}

The present study investigated the impact of statin therapy on midterm mortality after TAVI using a Japanese multicentre registry. Statin therapy at admission was associated with significantly lower all-cause and cardiovascular mortality. It should be noted that the impact of statin therapy attenuated in the nonagenarians. Furthermore, we demonstrated differences in all-cause mortality according to history of CAD and statin therapy. The present study included the largest patient cohort (936 pairs of patients after PS matching) and is the first report to investigate the association of age and history of CAD with the impact of statin.

Few reports have assessed the impact of statin treatment on mortality after TAVI. Peri-Okonny $e t a l^{7}$ demonstrated that statin therapy was associated with reductions in 2-year all-cause (aHR $0.65,95 \%$ CI 0.49 to $0.87, \mathrm{p}=0.001$ ), cardiovascular (aHR $0.66,95 \%$ CI 0.46 to $0.96, \mathrm{p}=0.030$ ) and non-cardiovascular (aHR 0.64, 95\% CI 0.44 to 0.99 , $\mathrm{p}=0.045$ ) mortality compared with no statin therapy, with a large cohort using PARTNER II and Sapien 3 clinical trials or associated registries (626 pairs of patients after PS matching). Merdler et $a l^{15}$ showed that high-intensity statin therapy was associated with a reduction in mortality after TAVI (median follow-up period: 2.5 years) using data of 1238 cases from a single-centre registry (aHR $0.59,95 \%$ CI 0.37 to $0.96, p=0.03$ ). Huded $e t a l^{16}$ also showed that high-intensity statin therapy was associated with a reduction in all-cause mortality (mean survival: 3.9 years) based on 294 cases (aHR 0.36, 95\% CI 0.14 to 0.90 , $\mathrm{p}=0.029$ ). Takagi et $a l^{17}$ reported similar results following a meta-analysis. These results were consistent with our results.

The mechanism through which statin therapy reduces the risks of all-cause and cardiovascular mortality is thought to be associated with a reduction in ischaemic events. ${ }^{75-17}$ 
However, there are limited data relative to statin therapy in octogenarians and nonagenarians, as are data not only on TAVI but also on statin therapy as primary and secondary prevention. The PROspective Study of Pravastatin in the Elderly at Risk trial was the only randomised controlled trial for elderly patients (aged 70-82 years) with history or risk factors of vascular disease. The study revealed that pravastatin led to a 3-year reduced risk of $\mathrm{CAD} .{ }^{18}$ Recommendation of statin therapy for very elderly patients varies among the guidelines. ${ }^{19-23}$ Very recently, a few reports supporting statin therapy for very elderly patients have been published. In the Patient and Provider Assessment of Lipid Management Registry, statin therapy appeared to be similarly tolerated by patients older and younger than 75 years. ${ }^{24}$ The Cholesterol Treatment Trialists' Collaboration demonstrated that statin therapy as primary and secondary prevention produced significant reductions in major vascular events even in patients older than 75 years. ${ }^{25}$ Furthermore, Ramos $e t a l^{26}$ revealed that statin therapy was associated with significant reductions in atherosclerotic cardiovascular events and all-cause mortality for patients who were older than 74 years and had diabetes. Interestingly, Giral et $a l^{27}$ demonstrated that statin discontinuation in 75-year-old primary prevention patients was associated with a $33 \%$ increased risk of cardiovascular events. Our present study was consistent with these reports and indicated that statin therapy would be effective for very elderly and atherosclerotic high-risk patients by reducing cardiovascular events and mortality. Conversely, Ramos $e t a t^{26}$ reported that the benefits of statin therapy disappeared in nonagenarians, as observed in our nonagenarian cohort. However, the $p$ value for interaction among the octogenarian and nonagenarian cohorts in the present study was not significant. There seemed to be a difference among the two cohorts during 12-24 months after TAVI but then the curves converged. The insignificance might be due to low life expectancy in nonagenarians after 24 months. Besides, the sizes of the cohorts and confounding regarding prescribing statin to nonagenarians with $\mathrm{CAD}$ might skew the results.

The statin effect generally appears after 1 year compared with placebo. ${ }^{728}$ Patients with a history of CAD who did not receive statin therapy appeared to have higher mortality rates after 1 year in the present study. In addition, our analysis combining history of CAD and statin therapy implied that TAVI patients with previous CAD might be able to achieve a similar reduction in mortality risk as those patients who had no previous $\mathrm{CAD}$ or statin treatment.

The present study had some limitations. First, this is an observational study, and unknown and unmeasurable factors may have confounded the relationship between statin therapy and mortality. However, a multicentre approach enabled us to accumulate a relatively large number of patients, and we used PS matching analysis, IPTW model and Cox proportional hazards regression model to confirm the robustness of the results. Second, a generalisation of the present results may be slightly limited due to the differences between the matched and unmatched group as it might be plausible given the results of the IPTW model. In addition, generalising our findings outside Japan also requires attention since this study included only Japanese patients. Third, information on the type and doses of statin therapy was not obtained. Use of ezetimibe or proprotein convertase subtilisin/kexin type 9 inhibitor was not recorded in this study. Fourth, we assessed statin use only on admission and there was a possibility that statin therapy might have changed at discharge or during follow-up. The duration of statin administration and the timing to start prescribing statin were not captured in the present study. Finally, we could not assess intolerance in patients eligible for statin treatment but who could not continue treatment due to statin side effects such as rhabdomyolysis. Further studies, including a randomised controlled trial, on statin therapy following TAVI are warranted to resolve these limitations.

In conclusion, using data from a large multicentre registry, statin therapy at admission for TAVI was associated with significant reductions in midterm all-cause and cardiovascular mortality. Statin therapy prior to TAVI will be beneficial even in octogenarians, but the benefits may disappear in nonagenarians. In addition, statin therapy will be essential for TAVI patients with CAD. Further research is warranted to confirm and generalise our findings since the present study has several inherent limitations of an observational study and included only Japanese patients.

\section{Author affiliations}

${ }^{1}$ Department of Cardiology, Saiseikai Utsunomiya Hospital, Utsunomiya, Japan

${ }^{2}$ Department of Cardiology, Keio University School of Medicine, Shinjuku-ku, Japan ${ }^{3}$ Center for Community-based Healthcare Research and Education, Shimane University Faculty of Medicine, Izumo, Japan

${ }^{4}$ Division of Cardiology, Vancouver General Hospital, Vancouver, British Columbia, Canada

${ }^{5}$ Department of Diagnostic Radiology, Keio University School of Medicine, Shinjukuku, Japan

${ }^{6}$ Department of Cardiovascular Surgery, Keio University School of Medicine, Shinjuku-ku, Japan

${ }^{7}$ Department of Cardiology, Toyohashi Heart Centre, Toyohashi, Japan

${ }^{8}$ Department of Cardiology, Nagoya Heart Centre, Aichi, Japan

${ }^{9}$ Department of Cardiology, Teikyo university school of medicine, Itabashi-ku, Japan

${ }^{10}$ Department of Cardiology, New Tokyo Hospital, Matsudo, Japan

${ }^{11}$ Department of Cardiology, Kokura Memorial Hospital, Kitakyushu, Japan

${ }^{12}$ Department of Cardiology, Saiseikai Yokohama City Eastern Hospital, Yokohama, Japan

${ }^{13}$ Department of Cardiology, Sendai Kousei Hospital, Sendai, Japan

${ }^{14}$ Department of Cardiology, Shonan Kamakura General Hospital, Kamakura, Japan

${ }^{15}$ Department of Cardiology, Osaka City General Hospital, Osaka, Japan

${ }^{16}$ Department of Cardiology, Toyama University Hospital, Toyama, Japan

${ }^{17}$ Department of Cardiovascular Surgery, Tokyo Bay Urayasu Ichikawa Medical

Centre, Urayasu, Japan

${ }^{18}$ Department of Cardiology, Ogaki Municipal Hospital, Ogaki, Japan

Twitter Taku Inohara @taku_inohara

Acknowledgements We express our heartfelt gratitude to the team members at our hospital and other hospitals participating in the multicentre registry and the Japan Society of Clinical Research (http://www.japanscr.org/) for their dedicated support.

Collaborators OCEAN-TAVI registry

Contributors Conception or design of the work: FYas, SS, TN, KM, MYamaw, MTab and FYam. Data collection: MTan, MJ, HS and KF. Data analysis and interpretation: $\mathrm{MH}, \mathrm{TI}, \mathrm{KT}, \mathrm{HU}$ and NT. Drafting the article: FYas and TI. Critical revision of the article: MYamam, YW and KH. Final approval of the version to be published: all authors. 
Funding The OCEAN-TAVI registry is supported by Edwards Lifesciences, Medtronic, Boston Scientific, Abbott Medical and Daiichi Sankyo Company. There are no award/grant numbers for the funders.

Competing interests MYamam, NT, TN, SS, KM, MTab, HU and YW are clinical proctors for Edwards Lifesciences and Medtronic. HS, KT and KH are clinical proctors for Edwards Lifesciences. TI received a research grant from Boston Scientific.

Patient consent for publication Parental/guardian consent obtained.

Ethics approval The institutional review boards of all the participating centres approved this study. Additionally, this study was conducted in accordance with the ethical guidelines of the 1975 Declaration of Helsinki.

Provenance and peer review Not commissioned; externally peer reviewed.

Data availability statement Data are available upon reasonable request. Please contact the corresponding author: yashima.fumiaki@gmail.com.

Supplemental material This content has been supplied by the author(s). It has not been vetted by BMJ Publishing Group Limited (BMJ) and may not have been peer-reviewed. Any opinions or recommendations discussed are solely those of the author(s) and are not endorsed by BMJ. BMJ disclaims all liability and responsibility arising from any reliance placed on the content. Where the content includes any translated material, BMJ does not warrant the accuracy and reliability of the translations (including but not limited to local regulations, clinical guidelines, terminology, drug names and drug dosages), and is not responsible for any error and/or omissions arising from translation and adaptation or otherwise.

Open access This is an open access article distributed in accordance with the Creative Commons Attribution Non Commercial (CC BY-NC 4.0) license, which permits others to distribute, remix, adapt, build upon this work non-commercially, and license their derivative works on different terms, provided the original work is properly cited, appropriate credit is given, any changes made indicated, and the use is non-commercial. See: http://creativecommons.org/licenses/by-nc/4.0/.

ORCID iD

Fumiaki Yashima http://orcid.org/0000-0002-7755-5771

\section{REFERENCES}

1 Mack MJ, Leon MB, Smith CR, et al. 5-year outcomes of transcatheter aortic valve replacement or surgical aortic valve replacement for high surgical risk patients with aortic stenosis (partner 1): a randomised controlled trial. Lancet 2015;385:2477-84.

2 Kapadia SR, Leon MB, Makkar RR, et al. 5-year outcomes of transcatheter aortic valve replacement compared with standard treatment for patients with inoperable aortic stenosis (partner 1): a randomised controlled trial. Lancet 2015;385:2485-91.

3 Popma JJ, Deeb GM, Yakubov SJ, et al. Transcatheter aortic-valve replacement with a self-expanding valve in low-risk patients. $N$ Engl J Med 2019;380:1706-15.

4 Mack MJ, Leon MB, Thourani VH, et al. Transcatheter aortic-valve replacement with a balloon-expandable valve in low-risk patients. $N$ Engl J Med 2019;380:1695-705.

5 Chakos A, Wilson-Smith A, Arora S, et al. Long term outcomes of transcatheter aortic valve implantation (TAVI): a systematic review of 5-year survival and beyond. Ann Cardiothorac Surg 2017;6:432-43.

6 Holmes DR, Brennan JM, Rumsfeld JS, et al. Clinical outcomes at 1 year following transcatheter aortic valve replacement. JAMA 2015;313:1019-28.

7 Peri-Okonny PA, Liu Y, Malaisrie SC, et al. Association of statin use and mortality after transcatheter aortic valve replacement. $J \mathrm{Am}$ Heart Assoc 2019;8:e011529.

8 Shimura T, Yamamoto M, Kano S, et al. Impact of the clinical frailty scale on outcomes after transcatheter aortic valve replacement. Circulation 2017;135:2013-24.

9 Yashima F, Yamamoto M, Tanaka M, et al. Transcatheter aortic valve implantation in patients with an extremely small native aortic annulus: the OCEAN-TAVI registry. Int J Cardiol 2017;240:126-31.
10 Kataoka A, Watanabe Y, Kozuma K, et al. Prognostic impact of low-flow severe aortic stenosis in small-body patients undergoing TAVR: the OCEAN-TAVI registry. JACC Cardiovasc Imaging 2018;11:659-69.

11 Kappetein AP, Head SJ, Généreux P, et al. Updated standardized endpoint definitions for transcatheter aortic valve implantation: the valve academic research Consortium-2 consensus document. J Am Coll Cardiol 2012;60:1438-54.

12 Rosenbaum PR, Rubin DB. The central role of the propensity score in observational studies for causal effects. Biometrika 1983;70:41-55.

13 Weitzen S, Lapane KL, Toledano AY, et al. Principles for modeling propensity scores in medical research: a systematic literature review. Pharmacoepidemiol Drug Saf 2004;13:841-53.

14 Austin PC. The performance of different propensity score methods for estimating marginal hazard ratios. Stat Med 2013;32:2837-49.

15 Merdler I, Rozenbaum Z, Finkelstein A, et al. Effect of statin therapy and long-term mortality following transcatheter aortic valve implantation. Am J Cardiol 2019;123:1978-82.

16 Huded CP, Benck LR, Stone NJ, et al. Relation of intensity of statin therapy and outcomes after transcatheter aortic valve replacement. Am J Cardiol 2017;119:1832-8.

17 Takagi H, Hari Y, Nakashima K, et al. Meta-analysis for impact of statin on mortality after transcatheter aortic valve implantation. Am J Cardiol 2019;124:920-5.

18 Shepherd J, Blauw GJ, Murphy MB, et al. Pravastatin in elderly individuals at risk of vascular disease (prosper): a randomised controlled trial. Lancet 2002;360:1623-30.

19 Mortensen MB, Falk E. Primary prevention with statins in the elderly. J Am Coll Cardiol 2018;71:85-94.

20 Piepoli MF, Hoes AW, Agewall S, et al. 2016 European Guidelines on cardiovascular disease prevention in clinical practice: The Sixth Joint Task Force of the European Society of Cardiology and other societies on cardiovascular disease prevention in clinical practice (constituted by representatives of 10 societies and by invited experts) developed with the special contribution of the European Association for Cardiovascular Prevention \& Rehabilitation (EACPR). Eur Heart $J$ 2016;37:2315-81.

21 US Preventive Services Task Force, Bibbins-Domingo K, Grossman $\mathrm{DC}$, et al. Statin use for the primary prevention of cardiovascular disease in adults: US preventive services Task force recommendation statement. JAMA 2016;316:1997-2007.

22 Anderson TJ, Grégoire J, Pearson GJ, et al. 2016 Canadian cardiovascular Society guidelines for the management of dyslipidemia for the prevention of cardiovascular disease in the adult. Can J Cardiol 2016;32:1263-82.

23 National Clinical Guideline Centre. Lipid modification: cardiovascular risk assessment and the modification of blood lipids for the primary and secondary prevention of cardiovascular disease. London, UK: National Institute for Health and Care Excellence, 2014.

24 Nanna MG, Navar AM, Wang TY, et al. Statin use and adverse effects among adults $>75$ years of age: insights from the Patient and Provider Assessment of Lipid Management (PALM) registry. J Am Heart Assoc 2018;7:e008546.

25 Cholesterol Treatment Trialists' Collaboration. Efficacy and safety of statin therapy in older people: a meta-analysis of individual participant data from 28 randomised controlled trials. Lancet 2019;393:407-15.

26 Ramos R, Comas-Cufí M, Martí-Lluch R, et al. Statins for primary prevention of cardiovascular events and mortality in old and very old adults with and without type 2 diabetes: retrospective cohort study. BMJ 2018;362:k3359.

27 Giral P, Neumann A, Weill A, et al. Cardiovascular effect of discontinuing statins for primary prevention at the age of 75 years: a nationwide population-based cohort study in France. Eur Heart $J$ 2019;40:3516-25.

28 Scandinavian Simvastatin Survival Study Group. Randomised trial of cholesterol lowering in 4444 patients with coronary heart disease: the Scandinavian simvastatin survival study (4S). Lancet 1994;344:1383-9. 